even broader class of structured spacetime beams.

Published online: 26 June 2020

$\square$ https://doi.org/10.1038/s41566-020-0652-7

References

Vincent Ginis (D) 1,2凶

${ }^{1}$ Data Lab/Applied Physics, Vrije Universiteit Brussel, Brussels, Belgium. ${ }^{2}$ Harvard John A. Paulson School of Engineering and Applied Sciences, Harvard University, Cambridge, MA, USA.

$\bigotimes_{e-m a i l: v i n c e n t . g i n i s @ v u b . b e}$
1. Kondakci, H. E. \& Abouraddy, A. F. Nat. Photon. 11, 733-740 (2017).

2. Kondakci, H. E. \& Abouraddy, A. F. Nat. Commun. 10, 929 (2019).

3. Donnelly, R. \& Ziolkowski, R. W. Proc. R. Soc. Lond. Ser. A 440, 541-565 (1993)
4. Saari, P. \& Reivelt, K. Phys. Rev. E 69, 036612 (2004).

5. Bhaduri, B., Yessenov, M. \& Abouraddy, A. F. Nat. Photon. https://doi.org/10.1038/s41566-020-0645-6 (2020).

6. Donnelly, R. \& Power, D. IEEE Trans. Antennas Propag. 45, 580-591 (1997)

7. Yu, N. et al. Science 334, 333-337 (2011)

8. Vezzoli, S. et al. Phys. Rev. Lett. 120, 043902 (2018).

9. Liberal, I. \& Engheta, N. Nat. Photon. 11, 149-158 (2017).

10. Rubinsztein-Dunlop, H. et al. J. Opt. 19, 013001 (2016)

\title{
BIOLUMINESCENCE
}

\section{Plants glow as they grow}

Plants that have been genetically engineered to glow bright green can provide visual signals to their state of health and development (T. Mitiouchkina et al., Nat. Biotechnol. https://doi.org/10.1038/ s41587-020-0500-9; 2020). In the future, once optimized, the functionality could lead to new imaging opportunities for monitoring and screening of plants as they grow.

Bioluminescence is a widespread phenomenon in the animal kingdom, with the firefly and some species of jellyfish, fish and octopi being common examples. While the feature has also been successfully introduced into plants by using bioluminescent genes and genetic engineering the light emission is usually too weak to be useful for applications. Now a team of scientists from Russia, the UK and Austria has managed to create genetically engineered plants that glow many times brighter than previous attempts. Indeed, the green glow from their tobacco plants is visible to the naked eye and reaches $10^{10}$ photons per minute, allowing it to be imaged using consumer-grade digital cameras rather than expensive, luminescent imaging equipment.

"We have established the feasibility of using fungal bioluminescence genes to produce glowing plants that are at least an order of magnitude brighter than was previously achieved using

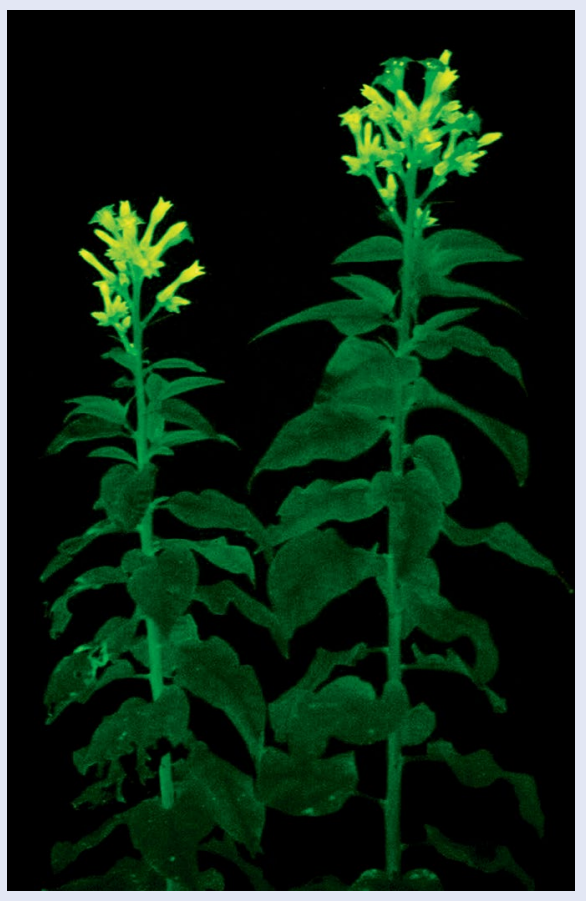

Credit: Springer Nature Ltd

a bacterial bioluminescence system," comment the researchers in their Nature Biotechnology paper. "By enabling autonomous light emission, dynamic processes in plants can be monitored, including development and pathogenesis, responses to environmental conditions and effects of chemical treatment."

Importantly, the fungal bioluminescence is driven by caffeic acid - a metabolic cycle in plants linked to the phenylpropanoid pathway that generates a rich source of metabolites such as flavonoids, coumarins, and lignins associated with healthy plant growth.

Indeed, the researchers' experiments indicated that the glow was brightest in certain parts of the plants associated with growth activity, specifically the flowers, the tips of the roots and cotyledons during seed germination, at sites of injury and also buds from young shoots.

"Roots glowed brightly at branching points, often hours before visible evidence of lateral root initiation," say the researchers. "Young shoots were brightest at the terminal and axillary buds and at the upper part of the stem; older parts of the shoots dimmed as plants matured."

The research was carried out by a team of 27 scientists, most of them affiliated to the Shemyakin-Ovchinnikov Institute of Bioorganic Chemistry in Moscow and Planta, a Moscow-based start-up founded in 2017 that is aiming to commercialize novel bioluminescent technologies.

Oliver Graydon

Published online: 26 June 2020

https://doi.org/10.1038/s41566-020-0661-6 